\title{
Los Programas Educativos para Parejas y Matrimonios: una nueva propuesta clínica para Chile
}

\section{Educational Programs for couples and marriages: a new clinical proposal to Chile}

\author{
Diana Rivera \\ Escuela de Psicología, Pontificia Universidad Católica de Chile \\ Patricio Cumsille \\ Escuela de Psicología, Pontificia Universidad Católica de Chile \\ Carmen Domínguez \\ Facultad de Derecho, Pontificia Universidad Católica de Chile. \\ Carmen Gloria Hidalgo \\ Escuela de Psicología, Pontificia Universidad Católica de Chile
}

(Rec: 13 marzo 2014 / Acept: 26 febrero 2015)

Resumen

\begin{abstract}
La educación dirigida a parejas y matrimonios tiene un amplio desarrollo en el mundo y en varios países se encuentra incorporada como parte de las políticas públicas preventivas de la discordia, ruptura y establecimiento de relaciones de parejas saludables y comprometidas. Iniciativas que originalmente fueron promovidas por grupos religiosos, hoy constituyen una alternativa preventiva y de promoción de relaciones saludables, basada en los avances de las investigaciones psicológicas y evaluadas en forma rigurosa. El artículo tiene por objetivo: (a) dar a conocer la lógica que fundamenta las intervenciones preventivas (b) dar a conocer las iniciativas en educación para parejas y matrimonios en otros países (c) sistematizar los hallazgos acerca de su efectividad (d) argumentar las razones por las cuales sería provechoso incorporarlas en el país.

Palabras Clave: educación para parejas, prevención, divorcio.
\end{abstract}

\section{Abstract}

Education aimed at couples and marriage has a broad development in the world and in several countries has been incorporated into adult educational programs as part of preventive public policies of disharmony, rupture and relationship healthy couples. Initiatives that were originally promoted by religious groups today are a preventive alternative to promote healthy relationships, based on advances in psychological research and rigorously evaluated. The paper presents (a) to present the logic behind preventive interventions (b) make known the initiatives in education for couples and families in other countries (c) systematize the findings about their effectiveness (d) discuss the reasons which would be useful for incorporation into the country. Key words: premarital education, prevention, divorce, engagement 


\section{Introducción}

En las últimas décadas del siglo XX, las familias han cambiado vertiginosamente más que en cualquier época tanto en su estructura, composición y función (Arriagada, 2002). En la esfera de las relaciones de pareja se constata un retraso en la edad de establecer una relación conyugal, aumento de las convivencias, disminución de las tasas de natalidad, aumento de los nacimientos fuera del matrimonio, aumento de rupturas, divorcios y rematrimonios (Instituto Nacional de Estadísticas [INE], 2012; United Nations [UN], 2011). También se producen cambios en las legislaciones (e.g., cuidado compartido de los hijos, homologación de los hijos ante la ley) y en varios países se amplían el vínculo legal marital a las uniones homosexuales (Países Bajos, Bélgica, España, Canadá, Sudáfrica, Noruega, Suecia, Portugal, Islandia, Uruguay, Argentina y Dinamarca). Todos lo anterior evidencia cambios sustantivos en los patrones de formación, ruptura y reconstitución de una relación de pareja estable y de la parentalidad.

Las rupturas de pareja y el divorcio, traen aparejado una serie de efectos negativos para el bienestar de las personas -adultos y niños- y grandes costos económicos y sociales para los gobiernos y la sociedad en su conjunto (Amato y Booth, 2001). También hay efectos beneficiosos, como el término de relaciones conyugales donde existe violencia intrafamiliar, abusos y malos tratos, y donde la insatisfacción marital sostenida deteriora la salud mental y física de las personas (Gottman, 1994, 1995). También se ha constatado que no sólo las rupturas provocan efectos adversos, sino también las relaciones de pareja estables pero infelices, pues los adultos presentan mayor morbilidad y mortalidad por alteraciones en el sistema cardiovascular, endocrino e inmune (Gottman, 1995; Robles y Kiecolt-Glaser, 2003). En el caso de los niños que pertenecen a familias donde los padres tienen altos niveles de conflicto, presentan más problemas de ajuste psicosocial (Amato et al., 2001; Morrison y Coiro, 1999) y más problemas en su salud física (Troxel, y Matthews, 2004) en comparación con niños de padres separados o con bajos niveles de conflicto.

Cuando se presentan altos niveles de conflictividad e insatisfacción con la relación, la psicoterapia de pareja es una de las alternativas de intervención. Sin embargo, estas parejas mayoritariamente no solicitan ayuda de ningún tipo (Johnson, Stanley, Glenn, Amato, Nock, Markman, et al., 2002) y cuando lo hacen, esperan en promedio más de seis años estando ya seriamente conflictuada y con altos niveles de insatisfacción (Christensen, 1999; Notarius y Buongiorno, 1992, citado en Gottman y Gottman, 1999).
A partir de mediados de los años 60' el incremento sistemático de las rupturas conyugales, de los hogares uniparentales surgidos por embarazos no planificados y los efectos adversos de esto en los niños y niñas, impulsó a los gobiernos de países occidentales desarrollados, como Estados Unidos, Australia, Noruega e Inglaterra, a considerar la institución del matrimonio como objeto de política pública. Una de las alternativas, fue la implementación de programas de educación premarital y de educación de adultos (e.g., apoyo a mujeres víctimas de violencia intrafamiliar, apoyo para un divorcio saludable, programas de mediación, programas de apoyo a la parentalidad). Estos programas estaban orientados a fortalecer la relación de pareja y un contexto familiar propicio para el desarrollo saludable de los niños y niñas (Helskog, 2009; van Acker, 2008).

Estas iniciativas generaron gran aceptación entre profesionales, investigadores y miembros de comunidades religiosas y seculares que, por diversas razones, compartían el interés por la promoción de relaciones maritales y de pareja estables y satisfactorias. Del mismo modo, también se generaron críticas, especialmente por la eventual injerencia del estado en la vida privada de las personas (Ooms, 2002).

La década de los 80 ' y 90', trajo consigo un desarrollo significativo de la investigación psicológica, lo que permitió determinar variables que caracterizaban a los matrimonios satisfechos e insatisfechos, estableciéndose con mayor claridad los factores protectores y de riesgo para la estabilidad y el bienestar de la relación (Gottman y Levenson, 2002; Kurdeck, 2002). Estos hallazgos fueron incorporados en los programas educativos y la evaluación de sus resultados fue paulatinamente cobrando mayor vigor y rigurosidad (e.g., Mathematica Policy Research, 2013; Office of Planning, Research y Evaluation [OPRE], 2013).

En Chile y Latinoamérica no existen programas de prevención y promoción de relaciones saludables para parejas y matrimonios, basados en la evidencia y probados en su efectividad. En el país -al igual que en otros casoshan sido los grupos religiosos los que han proporcionado orientación y guía a las parejas que van a iniciar una relación matrimonial, a las que desean enriquecer su relación y aquellas que se encuentran en conflicto (e.g., Encuentros Matrimoniales, Retrouville).

Actualmente en Chile se están desarrollando dos iniciativas investigativas en educación para parejas. La primera (Rivera, Cumsille, y Domínguez, 2012-2014), cuyo objetivo es adaptar y probar uno de los programas con mayor trayectoria y de probada eficacia, denominado Programa de Prevención y Enriquecimiento de las Relaciones (Prevention and Relationship Enhancement Program [PREP], Markman, 
Stanley y Blumberg, 1994), y la segunda, destinada a evaluar la viabilidad de su implementación como política pública (Hidalgo, Domínguez, y Rivera, 2011-2012). Ambas investigaciones están orientadas por el interés de asegurar que las intervenciones destinadas a las parejas y matrimonios, sean de la mejor calidad posible, basadas en conocimientos científicos e implementadas adecuadamente.

Los objetivos del artículo son (a) dar a conocer la lógica de las intervenciones preventivas en relaciones amorosas (b) dar a conocer las iniciativas en educación para parejas y matrimonios en otros países (c) sistematizar los hallazgos acerca de su efectividad (d) argumentar las razones por las cuales sería provechoso incorporarlas en el país.

\section{La prevención y los niveles de intervención}

Los programas dirigidos a parejas en relación de matrimonio o convivencia han sido denominados de muy diversas formas (e.g., programas de prevención, programa de enriquecimiento de la relación, promoción de matrimonios saludables, programas familiares) (Berger y Hannah, 1994). Esta nomenclatura ha generado una cierta confusión, pues en ella se mezclan objetivos, población meta y los enfoques y técnicas que emplean (Fincham, Stanley, y Rhoades, 2010). Una forma conveniente para entender las intervenciones, es según el nivel preventivo donde operan (Berger et al., 1999). Así, en un polo se ubica la prevención primaria, que incluye las intervenciones que acontecen antes que se susciten los problemas definidos como indeseables. En el caso de las parejas, el momento privilegiado de intervención sería previo a la vida conjunta, siendo representante de este tipo de intervenciones, la educación premarital y la preparación parental. Siguiendo en el continuo, existen programas de enriquecimiento de la relación de pareja unidas en vínculo legal o no, donde participan parejas sin problemas o bien con problemas más bien moderados, que pueden ser subsanados con un apoyo breve o intervenciones de baja complejidad. Estas intervenciones están destinadas a fomentar la satisfacción y el bienestar en las parejas, correspondiendo a intervenciones de prevención secundaria. Los programas remediales refieren a intervenciones cuyo objetivo es mitigar o eliminar las causas que se asocian con la aparición y/o mantención de problemas, donde las parejas se reconocen en conflicto, se encuentran insatisfechas y la disolución del vínculo es una posibilidad. En este caso, la psicoterapia es la modalidad más adecuada para ellas, o bien aquellas intervenciones que faciliten una separación o divorcio no destructivo (e.g., mediación), correspondiendo a prevención terciaria.
A lo largo de los años la unidad de intervención de los programas educativos ha ido ampliándose a personas, parejas y matrimonios. El objetivo de las intervenciones educativas, más que como se planteó en un comienzo - prevenir la ruptura marital o el divorcio- es reducir los factores de riesgo y su impacto negativo, de modo de incrementar la calidad de vida de los adultos y los niños, mediante acciones dirigidas hacia la sociedad y la pareja (Stanley, 2001). Así, los programas que proveen educación estructurada sobre las relaciones, actitudes y habilidades para las parejas, se han denominando educación para la relación de pareja (Halford, Markman, y Stanley, 2008; Halford, Markman, Kline, y Stanley, 2003). Últimamente Markman y Rhoades (2012) proponen omitir el término "pareja" de modo de incluir intervenciones donde pueden asistir en forma individual personas que tienen pareja y otras que no, pero que tienen interés en establecer y/o mantener relaciones saludables. Prefieren utilizar el término educación para las relaciones, que incluye tanto las acciones y los programas que proporcionan educación, desarrollo de habilidades y cuyos principios orientadores ayudan a las personas y a las parejas a aumentar la probabilidad de formar una relación estable y saludable.

Los factores protectores y de riesgo para la estabilidad y satisfacción de las relaciones de pareja y matrimonio, corresponden a diferentes niveles de complejidad: nivel individual, nivel de pareja, nivel de la familia de origen, y nivel contextual (ver Beach, Kamen, y Fincham, 2006; Karney y Bradbury, 2005). Los programas educativos para parejas y matrimonios, dirigen sus esfuerzos hacia los factores de riesgo que son relativamente dinámicos y modificables (Stanley, 2001), como por ejemplo aprender modalidades saludables de resolución de conflicto o hacerse consciente de expectativas maritales poco realistas que guían las interacciones y son fuente de frustración.

\section{La experiencia de otros países}

Este apartado se elaboró recogiendo la experiencia de cuatro países con trayectoria en la educación para familias, parejas y matrimonios, Estados Unidos, Australia, Inglaterra y Noruega.

\section{Objetivo de los programas}

La educación para parejas en Estados Unidos, ha estado focalizada predominantemente al fortalecimiento del matrimonio, mientras que en Australia e Inglaterra, esto es menos explícito y se dirige a personas unidas en matrimonio 
o cohabitación, e incluso para personas que serán padres pero no pareja, orientándose en este caso, fuertemente al bienestar de los niños (van Acker, 2008). En el caso de Noruega, explícitamente los programas mencionan la promoción de relaciones saludables y comprometidas, y no el matrimonio como tal. Estos énfasis obedecen a diferencias culturales, demográficas y visiones políticas más liberales o conservadoras respecto a la institución del matrimonio (van Acker, 2008). Estos aspectos son necesarios de considerar cuando se adaptan y aplican los programas a realidades distintas. Por ejemplo, en Noruega, el $90 \%$ de las parejas cohabita antes de casarse (Helskog, 2009). Coherentemente con lo anterior, la adaptación del programa Prevention Relationship and Enhacement Program (PREP) en Noruega consideró como objetivo del programa, el enriquecimiento de la relación de pareja y no la prevención del divorcio, y a su vez, el título del libro Fighting for your marriage (Markman, Stanley, y Blumberg, 1994) empleado como material de apoyo, se tradujo como El Libro para la Vida Conjunta (The book of living together) (Helskog, 2009). Aún cuando se constatan matices entre los países, en especial en Inglaterra donde el foco fundamental está en los niños y la parentalidad, independientemente del tipo de vínculo entre los padres, todos los gobiernos comparten la visión que, las relaciones saludables y comprometidas de pareja y las familias estables, son beneficiosas para los individuos y la sociedad en su conjunto (van Acker, 2003, 2008). A su vez, Halford y sus colegas (2008) destacan que la educación para parejas a lo largo de los años, ha ido abarcado además de parejas de novios, a parejas de larga data que buscan fortalecer su relación, o aquellas que se volverán a casar, o a quienes van a tener un hijo, y junto a lo anterior, han incorporado nuevos contextos de implementación (e.g., hospitales, cárceles).

\section{Las agencias que imparten educación para parejas}

En los países mencionados, la política pública de educación para parejas se desarrolla a través de la colaboración entre el estado y diversas organizaciones e instituciones de la sociedad civil, quienes postulan a los fondos para implementar las intervenciones. Cada país tiene formas de seleccionar a las agencias que imparten educación, las cuales son muy variadas en tamaño, radio de acción y organización entre ellas. A modo de ejemplo, en Australia el estado exige que los programas deben estar basados en los objetivos y lineamientos establecidas por el Programa de Servicios para las Relaciones Familiares (Family Relationships Service Program, FRSP), deben tener como objetivo impartir educación a hombres y mujeres para que desarrollen e incrementen habilidades para construir relaciones positivas y estables con la pareja o la familia, deben adherir a un enfoque centrado en la prevención, compartir los preceptos de la educación de adultos y proporcionar entrenamiento en habilidades relacionales (van Acker, 2008). En el caso de Estados Unidos, se plantea un mínimo de horas de intervención.

Por otra parte, los montos de financiamiento asignado a este tipo de programas, depende de las políticas y orientaciones de los gobiernos en ejercicio (Halford y Simons, 2005; Ooms, 2002; van Acker, 2008). Los fondos pueden provenir del gobierno central, de fondos de cada estado, de las iglesias, de centros jurídicos de la comunidad, de organizaciones comunitarias independientes, redes de las Iglesias, o de otros benefactores que suman esfuerzos para implementar las intervenciones. En general, los gobiernos a través de sus distintos departamentos proporcionan fondos de tipo operativo y otros por pago por del servicio prestado; algunas organizaciones cobran a los usuarios pequeños montos en base al ingreso familiar, pero básicamente son los gobiernos quienes subvencionan la mayor parte de estas actividades (Ooms, 2002, 2005; van Acker, 2008). Además se producen asociaciones entre centros privados y universidades lo cual permite evaluar los programas implementados (e.g., Instituto Relate y la Universidad de Hull en Inglaterra; PREP y la Universidad de Denver).

\section{Los programas educativos de prevención y promoción}

Los programas de educación para parejas y matrimonios tienen una larga trayectoria y se han ido modificando a lo largo de los años. Tienen diferencias en sus marcos conceptuales, en la duración y frecuencia de las sesiones y en las modalidades de implementación. Varios textos describen en forma detallada los programas más relevantes (ver Berger et al., 1999; Dion, 2005, 2006; Silliman y Schumm, 2000). Por lo general, la gran mayoría de ellos trabajan con ambos miembros de la pareja, en sesiones de grupo, lideradas por uno o dos educadores entrenados, en sesiones impartidas semanalmente $\mathrm{y} / \mathrm{o}$ los fines de semana, y dirigidos a parejas heterosexuales. Habitualmente tienen una duración promedio de 12 horas. Paulatinamente se han incorporado nuevos contenidos que basados en la investigación, informan que favorecen la relación (e.g., compromiso, generosidad, perdón, apoyo y vínculo seguro) y nuevas poblaciones objetivo, como personas y parejas en estados iniciales de compromiso, parejas de sectores desventajados, entre 
otras (ver Markman et al., 2006). Por lo general tienen un grado de estructuración media a alta, emplean materiales como videos, cuestionarios, y son didácticos y vivenciales (Berger et al).

Por motivos de espacio sólo se describirán dos programas que evidencian tendencias distintas dentro de la gran gama de opciones existentes: el Programa PREPARE/ENRICH que corresponde a la línea de intervenciones basadas en la evaluación de la pareja y entrega de feedback y el programa PREP con un currículo basado en la enseñanza de conocimientos y habilidades específicas.

PREPARE/ENRICH: Premarital Preparation and Relationship Questionnaire (PREPARE). Este programa fue diseñado originalmente por el investigador David Olson en 1978 y ha tenido tres revisiones posteriores. Su premisa fundamental es que la calidad de la relación marital se puede predecir a partir de la relación premarital, motivo por el cual su trabajo se desarrolla fundamentalmente con parejas comprometidas. Sostienen un enfoque teórico ecléctico y basado en estudios empíricos. El formato de intervención es de uno a uno, es decir se atiende a cada pareja individualmente de acuerdo a sus necesidades particulares. Los miembros de la pareja contestan vía on line un cuestionario de 165 itemes que evalúa las fortalezas y áreas a desarrollar en la relación. Las personas deben tener un grado educacional correspondiente a sexto grado para contestarlo adecuadamente. Se promueve el enriquecimiento de las habilidades comunicacionales, se identifican y discute cómo manejar de buena forma los principales estresores, a resolver los conflictos, a discutir el manejo de las finanzas, a establecer metas, y comprender y apreciar las diferencias de personalidad de cada uno de los miembros de la pareja. Su grado de estructuración es alto, y se desarrolla en un formato de 2 o 4 horas, donde después de completar el cuestionario, las parejas reciben retroalimentación de una persona debidamente entrenada. El modelo de facilitación es didáctico experiencial, se dan tareas y se ven videos. El cuestionario de evaluación tiene el valor de hacer más conscientes a las personas de su propia perspectiva y que su pareja la conozca. A su vez, proporciona al consejero información acerca del funcionamiento de la pareja, lo cual le permite orientar mejor la ayuda.

PREP: El programa PREP, fue creado por Markman, Stanley y Blumberg (1994), y se encuentra sólidamente fundamentado en la investigación empírica. Sus báses téoricas son la teoría del aprendizaje social y el enfoque cognitivo-conductual. Los temas centrales que aborda son la comunicación, la resolución de conflictos, habilidades de negociación, la reestructuración de expectativas, la promoción de la diversión, la sensualidad, la amistad, el compromiso y la relevancia de establecer una relación segura, en un contexto seguro. La duración del programas es de 6 sesiones de 2 horas cada una, o de un taller de fin de semana (2 días) o de 1 día de taller y dos tardes, lo cual completa 12 horas; también hay una versión abreviada de 8 horas denominada "A Nuestro Alcance" (Whithin our reach) de 8 horas de duración. El modelo de enseñanza es didáctico, experiencial, grupal y con trabajos individuales para ser conversados con la pareja; ocupa videos demostrativos y tareas escritas. Respecto de los facilitadores, éstos pueden ser profesionales, clérigos u otros líderes entrenados en PREP. Está dirigido a todo tipo de parejas, comprometidas, novios y matrimonios.

\section{La efectividad de los programas de prevención}

Un número importante de revisiones evidencia que la educación premarital en general es efectiva (Carroll y Doherty, 2003; Fagan, Patterson, y Rector, 2002; Halford et al., 2003; Silliman, Stanley, Coffin, Markman, y Jordan, 2002), aun cuando un número menor ha sido sistemáticamente evaluado (Halford, 1999; Stanley, 2001). Los primeros programas se focalizaron en la evaluación de comportamientos específicos, como habilidades comunicacionales, resolución de conflictos y otras que se asociaban con el buen funcionamiento de las relaciones de pareja (Knox y Fein, 2012). Los estudios realizados en estos programas denominados de primera generación, se caracterizaban por tener muestras pequeñas donde participaban fundamentalmente personas blancas educadas, desarrollados por clínicos e investigadores, siendo criticados por lo restringido del universo estudiado (Ooms y Wilson, 2004). Se han efectuado varios meta análisis considerando distinto tipo de intervenciones. Uno de ellos, incluyó 26 estudios de cursos de educación premarital, evaluados con diseños experimentales, cuasi experimentales, no experimentales y ex-post-facto, donde se encontró que los programas en general son efectivos con un tamaño del efecto de .80 (Carroll et al., 2003). Otros meta análisis realizados en educación para parejas, evidencian un tamaño del efecto moderado a alto en incrementar la comunicación en la pareja (Blanchard, Hawkins, Baldwin, y Fawcett, 2009) y un tamaño del efecto pequeño a moderado en la satisfacción con la relación, evaluada inmediatamente después de finalizada la intervención (Carroll et al., 2003; Hawkins et al., 2008). Un tercer meta análisis realizado por Hawkins, Blanchard, Baldwin y Fawcett (2008) consideró, 117 estudios que incluían intervenciones educativas de diverso tipo: educación para las relaciones impartida para personas solteras, para parejas seriamente comprometidas, 
programas de promoción dirigidos a novios y matrimonios, y otros para parejas en vías de ser padres. Los diseños utilizados eran experimentales, cuasi experimentales, evaluación pre post intervención y de seguimiento. Este meta análisis se centró en la evaluación de adquisición de habilidades comunicacionales y calidad de la relación, siendo el tamaño del efecto de .36 a .54 y .24 a .36 respectivamente.

Para responder a la interrogante y cuestionamiento de la falta de intervenciones y estudios con poblaciones vulnerables, Hawkins y Fackrell (2010) realizaron un meta análisis con 15 estudios donde participaban parejas y familias pobres, donde se emplearon diseños experimentales y no experimentales de evaluación. El resultado permitió establecer que producían un tamaño del efecto pequeño a moderado (.19 a .25) en la calidad de la relación y en habilidades comunicacionales (Hawkins y Fackrell, 2010).

En relación a la duración de los efectos de las intervenciones, hay antecedentes que evidencian que la efectividad de los programas dirigidos el mejoramiento de la calidad de la relación de pareja, muestran diferencias significativas con los grupos control después de $11 / 2$ año y 3 años posteriores a la intervención (Markman, Floyd, Stanley, y Storaasli, 1988) y en parejas en riesgo (Schilling, Baucom, Burnett, Allen, y Ragland, 2003). El programa PREP es uno de los programas que ha realizado evaluación de seguimiento de más largo plazo (Renick, Blumberg, y Markman, 1992; Markman, Rhodes, Stanley, Ragan, y Whitton, 2010; Stanley, Amato, Johnson, y Markman, 2006; Stanley, Rhoades, Olmos-Gallo, y Markman, 2007).

En la revisión de los estudios del año 2002-2009, Markman y Rhoades (2012) encontraron 30 estudios que evaluaban 21 programas. Destacan dos por el aporte que hacen a lo que ellos denominan como una nueva fase educativa en las relaciones interpersonales significativas: a) Aplicación del programa PREP en la milicia en Estados Unidos, por su aporte a la reducción del divorcio al doble de su valor inicial, evaluado a un año de la intervención (Stanley, Allen, Markman, Saiz, Bloomstrom, Walter, et al, 2005) b) Programa desarrollado en el estado de Oklahoma, por su efectividad en la reducción de las rupturas de pareja y mejoramiento en indicadores de calidad de las relaciones y por su aporte en la introducción de nuevas metodologías (ver Devaney y Dion, 2010).

Un hallazgo adicional de estos programas, son los efectos beneficiosos en las prácticas parentales, en la relación del padre con el niño (Carlson y McLanahan 2006) y cuando los niños se encuentran en la edad de incorporarse al sistema escolar (Cowan, Cowan, y Heming, 2005). La relación entre parentalidad y la relación de pareja, es un ámbito que requiere de mayor investigación y estudios longitudinales (Cowan et al.).

\section{La realidad en Chile y aportes potenciales al país}

Los patrones de conformación de la relación de pareja en Chile y la parentalidad, se van asemejando a la realidad de países desarrollados. Los últimos antecedentes sobre fecundidad y familia (INE, 2012), evidencian un aumento en la edad promedio para contraer matrimonio en primeras nupcias, correspondiendo en el caso de los hombres a 31.3 años y en las mujeres a 29.9 años. Previo al matrimonio, la soltería es el estado civil predominante de los cónyuges, $94.8 \%$ en el caso de las mujeres y de $92.6 \%$ para los varones. A su vez, muchas personas que legalizan su unión, tienen un hijo nacido fuera del matrimonio. De hecho, de 10 niños que nacen en Chile, 7 son hijos de padres que no están casados legalmente y el $14.9 \%$ de los nacidos, son hijos de madres adolescentes menores de 20 años. Estos datos evidencian que un número relevante de parejas entra a la vida conjunta con la exigencia de incluir un hijo de alguna relación anterior o de la misma pareja.

La mayoría de las personas en Chile, vive en familia, pues el $82.1 \%$ de los hogares están compuestos por personas en vínculo de parentesco, ya sea en hogares nucleares, familias extensas u hogares compuestos (INE, 2012). La conformación de una relación de pareja estable y de una familia es un anhelo para la mayoría de los jóvenes (INJUV, 2003). Sin embargo, a pesar de la valoración de la pareja, el matrimonio y de la familia, de acuerdo a los registro de Naciones Unidas (United Nations, 2011), en Chile el 19.2\% de los matrimonios se divorcia en los primeros cinco años de relación. Este porcentaje sería mayor si se incluyera a las parejas que conviven y luego se separan, y aquellas casadas que se separan de hecho y materializan la disolución legal del vínculo años más tarde. Evidentemente el hecho de conformar una relación de matrimonio o convivencia estable y una familia, no asegura de por sí, que estos vínculos sean satisfactorias y saludables. Los programas educativos para parejas y matrimonios pueden aportar en varios aspectos críticos de las parejas en el país, que se detallan a continuación.

Malos tratos y violencia: Existe una alta prevalencia de violencia intrafamiliar en el país (Ministerio del Interior, 2010). En el caso de los jóvenes entre 15 y 29 años, el $16 \%$ reporta haber experimentado violencia en su relación actual, la cual aumenta con la edad, llegando a un $21 \%$ en el tramo de 25 a 29 años, edad donde mayoritariamente las parejas conforman una relación estable. A su vez, la 
violencia es mayor entre los jóvenes que tienen al menos un hijo, pues el $24 \%$ declara haber experimentado alguna situación de violencia al interior de su actual relación de pareja (INJUV, 2013). La conflictividad muchas veces se extiende aún después de disuelta la relación a través de divorcios destructivos (Hamel, Bernales, Campos, Estrada, Gazmuri et al., 1997). Markman y Rhoades (2012) sugiere trabajar especialmente con personas y parejas jóvenes con distintos niveles de compromiso y formalización, de modo que favorecer tempranamente una mejor toma de decisiones, que en algunos casos puede llevar a posponer el compromiso o incluso terminarlo antes de su concreción y/o formalización. Los programas educativos al enseñar habilidades en la resolución pacífica de conflictos, promover habilidades de comunicación como el desescalamiento y el control emocional, pueden contribuir a disminuir la violencia al interior de la relación (Markman et al., 1993). Holtzworth-Munroe y sus colaboradores (1995) sugieren que los efectos de los programas premaritales en la prevención de la violencia de pareja, son mayores en población en riesgo. Estos hallazgos son relevantes de considerar, pues la agresión verbal y física ocurre tempranamente y es un predictor sustantivo del quiebre de la relación (Lawrence y Bradbury, 2001). En Chile se han implementado algunos programas de prevención con estudiantes universitarios pero de escasa cobertura (Póo y Vizcarra, 2010).

Parejas pertenecientes a población vulnerable y de riesgo: En los últimos años se han sido implementados exitosamente estos programas en población desventajadas (Ooms et al., 2004; Ooms, 2007), los cuales pueden también operar en el apoyo a la transición a la parentalidad (Petch, Halford, Creedy, y Gamble, 2012). En la aplicación del programa PREP masivamente en el estado de Oklahoma en Estados Unidos con familias pobres, se constató que la efectividad de estas intervenciones aumenta significativamente con sesiones de reforzamiento (Ooms, 2007).

En Chile, el sistema de salud está por muchos años en contacto permanente con los adultos, a través de la atención que provee a los niños. Esta es una instancia privilegiada de conocimiento y contacto con las parejas que pueden necesitar apoyo tanto respecto de la vida de pareja, como de la parentalidad.

Apoyo a la parentalidad: Tal como la literatura lo indica, estas intervenciones pueden promover una mejor parentalidad (Carlson et al., 2006; Cowan et al., 2005) lo cual es relevante en la medida que muchas parejas establecen una relación comprometida donde uno o ambos son padres. Las salas cuna y los Jardines Infantiles pueden ser espacios de promoción, difusión e implementación de los programas educativos para personas y parejas, basados en la motivación de los padres por desarrollar de mejor forma la parentalidad.

Enriquecimiento de matrimonios y parejas constituidas: Los programas también pueden beneficiar parejas establecidas en relación de matrimonio o convivencia que presenten niveles medios y bajos de conflictividad. Estas parejas y matrimonios saben de las complejidades de la vida conjunta y con intervenciones breves pueden fortalecer su relación (Schilling et al., 2003). Las parejas, matrimonios y familias transitan por distintos ciclos en los cuales es posible intervenir para promover relaciones saludables y satisfactorias entre sus miembros, reforzando y adaptando el entrenamiento a habilidades específicas de acuerdo al ciclo vital, como por ejemplo para las parejas en relación de reconstitución de pareja o re matrimonio (Renick et al., 1992). De hecho, en la aplicación del Programa PREP en el año 2013, un número relevante de parejas solicitaba participar de los talleres aunque no cumplían con uno de los criterios establecidos en esa ocasión, de mantener una relación de matrimonio o convivencia de hasta 5 años.

\section{Conclusiones}

Transcurridos más de treinta años de aplicación de programas de educación para parejas, se constata la efectividad de los mismos, una renovación sistemática para hacerlos cada vez más efectivos (e.g. sesiones de apoyo) y sometidos a evaluaciones cada vez más exigentes. Los cambios demográficos han llevado a que estos programas se ofrezcan no sólo a novios y matrimonios, sino también a parejas de convivientes, parejas re constituidas o en re matrimonio, y últimamente a personas sin pareja, lo que muestra el interés de personas en distintas situaciones por establecer relaciones estables y saludables, y el interés del estado por una mayor cobertura de sus políticas públicas.

La experiencia internacional de colaboración entre el estado y organizaciones sociales e instituciones de diverso tipo, a través del financiamiento de acciones de intervención y evaluación, permite que los gobiernos por una parte, materialicen sus políticas públicas y por otra parte, obtengan rápidamente información para la toma de decisiones, basadas en la evidencia y conociendo experiencias prácticas diversas, dada la cercanía privilegiada que tienen las organizaciones con la comunidad.

En Chile existen algunas iniciativas que se asemejan a la experiencia internacional, aunque en el campo de la prevención terciarias, específicamente en relación al traslado de fondos para la atención de familias donde hay violencia 
intrafamiliar (e.g., atención de hombres que han ejercido violencia; apoyo a mujeres víctimas de violencia intrafamiliar). Sin embargo hay mucho que avanzar en este camino pues aún los fondos son escasos y operan básicamente a nivel de la prevención terciaria.

Finalmente, es necesario destacar que estas acciones no son la panacea, pues la calidad y estabilidad de una relación de pareja y matrimonio, está determinada por múltiples factores. Los programas educativos intervienen a nivel de las dimensiones que las personas pueden modificar y que afectan la dinámica relacional en forma significativa. Sin embargo, hay otros niveles que están fuera de su alcance y que afectan la vida íntima de las personas, como son la pobreza, la desigualdad, la inseguridad, el estrés y situaciones propias de la vida moderna (Karney et al., 2005). En este sentido, desde una mirada ecológica, es necesario cuidar de colocar excesiva responsabilidad en las personas $\mathrm{y}$ no en las condicionantes estructurales que fragilizan a las familias y los vínculos entre las personas.

Es necesario que los profesionales de la salud mental puedan abrirse a implementar intervenciones de este tipo, ampliado su quehacer clínico más allá de la psicoterapia, en un trabajo conjunto con instituciones, organizaciones y servicios interesados en esta apasionante tarea.

\section{Referencias}

Amato, P. R., y Booth, A. (2001). The legacy of parents' marital discord: Consequences for children's marital quality. Journal of Personality and Social Psychology, 81, 627-638.

Arriagada, I. (2002). Cambios y desigualdad en las familias latinoamericanas, Revista de la CEPAL, 77, 143-16.

Beach, S. R., Kamen, Ch., y Fincham, F. (2006). Marital Dysfunction. En Frank Andrasik, (Editor) Comprehensive Handbook of Personality and Psychopathology (CHOPP) Volume II: Adult Psychopathology (pp. 450-468). New Jersey: John Wiley y Sons Inc.

Berger, R., y Hannah, M. T. (1999). Preventive Approaches in Couples Therapy. USA: Brunner /Mazel.

Blanchard, V. L., Hawkins, A. J., Baldwin, S. A., y Fawcett, E. B. (2009). Investigating the effects of marriage and relationship education on couples' communication skills: A meta-analytic study. Journal of Family Psychology, 23, 203-214.

Carroll, J. S., y Doherty, W. J. (2003). Evaluating the effectiveness of premarital prevention programs: A meta-analytic review of outcome research. Family Relations, 52, 105-118.

Carlson, M. J., y McLanahan, S. (2006). Strengthening unmarried families: Could enhancing couple relationships also improve parenting? Social Service Review, 80, 297-321.

Cowan C. P., Cowan, P. A., y Heming, G. (2005). Two variations of a preventive intervention for couples: Effects on parents and children during the transition to school. En P. A. Cowan, C. P. Cowan, H. Ablow, V. K. Johnson, y J. R. Measelle (edts), The family context of parenting in children's adaptation to elementary school (pp. 277-312). Mahwah, NJ: Lawrence Erlbaum Associates.

Christensen, A. (1999). "Interventions for Couples". Annual Review of Psychology, 50, 165-190.
Doherty, W. J., y Anderson, J. R. (2004). Community Marriage Initiatives. Family Relations, 53, 425-432.

Devaney, B., y Dion, R. (2010). 15-month impacts of Oklahoma's Family Expectations program. Princeton, NJ: Mathematica Policy Research. Recuperado desde http://www.mathematica-mpr.com/publications/ PDFs/Family_support/BSF_15month_impacts.pdf

Dion, R. M. (2005). Healthy Marriage Programs: Learning What Works, Future of Children, 15, 139-156.

Dion, R. M. (2006). Marriage Initiative: An Overview of the Longest Running Statewide Marriage Initiative in the US. Mathematica Policy Research Inc. Recuperado desde http://www.acf.hhs/healthymarriage.

Fagan, P. F., Patterson, R. W., y Rector, R. E. (2002). Marriage and Welfare Reform: The Overwhelming Evidence that Marriage Education Works. The Heritage Foundation. No. 1606.

Fincham, F. D., Stanley, S. M., y Rhoades, G. K. (2010). Relationship education in emerging adulthood: Problems and prospects. En F. D. Fincham y M. Cui (edts), Advances in Personal Relationships: Romantic relationships in emerging adulthood (pp. 293-316). Cambridge: Cambridge University Press.

Gottman, J. (1994). What Predict Divorce? The relationship between marital processes and marital outcomes. New Jersey: Lawrence Erlbaum Associates.

Gottman, J. (1995). The Seven Principles for Making Marriage Work. New York: Random House.

Gottman, J., y Gottman, J. (1999). The marriage survival kit. En R. Berger y M. T. Hannahh (eds), Preventive approaches in couples therapy (pp. 304-330). Philadelphia, PA: Brunner/Mazel.

Gottman, J. M., y Levenson, R. W. (2002). A two-factor model for predicting when a couple will divorce: Exploratory analyses using 14-year longitudinal data. Family Process, 41, 83-96.

Halford, W. K. (1999). Australian couples in Millenium three: A research and development agenda for marriage and relationship education. Report to the National Family Strategy Task Force, Australian Department of Family and Community Services. Brisbane, Australia: Australian Academic Press.

Halford, W., Markman, H., Kline, G., y Stanley, S. (2003). Best practice in couple relationship education. Journal of Marital and Family Therapy, 29, 385-406

Halford, W. K., Markman, H. J., y Stanley, S. (2008). Strengthening couples' relationships with education: Social policy and public health perspectives. Journal of Family Psychology, 22, 497-505.

Halford, W, K., y Simons, M. (2005). Couple Relationship Education in Australia. Family Process, 44, 147-159.

Hamel, P., Bernales, S., Campos, S., Estrada, A., Gazmuri, V., Navarro, M., y Ortiz de Zúñiga, B. (1997). Separación matrimonial: repercusiones y recursos. Resultados de una investigación. Revista De Familias y Terapias, 8, 37-53.

Hawkins, A. J., Blanchard, V. L., Baldwin, S. A., y Fawcett, E. B. (2008). Does marriage and relationship education work? A meta-analytic study. Journal of Counseling and Clinical Psychology, 76, 723-734.

Hawkins, A. J., y Fackrell, T. A. (2010). Does relationship and marriage education for lower income couples work? A meta-analytic study of emerging research. Journal of Couple y Relationship Therapy, 9, 181-191.

Helskog, G. H. (2009). The Norwegian state: A relationship educator. In $\mathrm{H}$. Benson and S. Callan (Eds.), What works in relationship education: Lessons from academics and service deliverers in the United States and Europe (pp. 137 - 148). Doha, Qatar: Doha International Institute for Family Studies and Development. House of Representatives Standing Committee on Legal and Constitutional Affairs, (HRSCLCA)

Hidalgo, C. G., Domínguez, C., y Rivera, D. (2011-2012). Proyecto Promoción de Políticas Públicas para el Fortalecimiento del Matrimonio. Vicerrectoría de Investigación (2011-2012) № 20/11.

Holtzworth-Munroe, A., Markman, H., O’Leary,K. D., Neidig, P., Leber, D., Heyman, R. E., Hulbert, D., y Smutzler, N. (1995). The need for marital violence prevention efforts: A behavioral-cognitive secondary prevention program for engaged and newly married couples. Applied and Preventive Psychology, 4, 77-88. 
Instituto Nacional de Estadísticas (2012). Estadísticas vitales. Informe Anual 2010. INE.

Instituto Nacional de la Juventud (2003). Cuarta Encuesta Nacional de la Juventud. Ministerio de Desarrollo Social. Gobierno de Chile.

Instituto Nacional de la Juventud (2013). 7ma Encuesta Nacional de Juventud 2012. Ministerio de Desarrollo Social. Gobierno de Chile.

Johnson, C. A., Stanley, S. M., Glenn, N. D., Amato, P., Nock, S. L., Markman, H. J., et al. (2002). Marriage in Oklahoma: 2001 baseline statewide survey on marriage and divorce (S02096 OKDHS). Oklahoma City: Oklahoma Department of Human Services.

Karney, B. R., y Bradbury, T. N. (2005). Contextual influences on marriage: Implications for policy and intervention. Current Directions in Psychological Science, 14, 171 - 174.

Knox, V., y Fein, D. (2012). Supporting Healthy Marriage. Designing a Marriage Education Demonstration and Evaluation for Low-Income Married Couples. En H. Peters, E. Dush, y C. Kamp (eds), M. Marriage and Family: Perspectives and Complexities (pp. 247-280). New York, NY, USA : Columbia University Press.

Kurdeck, L. A. (2002). Predicting the timing of separation and marital satisfaction: An eight-year prospective longitudinal study. Journal of Marriage and Family, 64, 163-179.

Lawrence, E., y Bradbury, T.N. (2001). Physical aggression and marital dysfunction: A longitudinal analysis. Journal of Family Psychology, $15,135-154$

Markman, H. J., Floyd, F. J., Stanley, S. M., y Storaasli, R. D. (1988). Prevention of Marital Distress: A Longitudinal Investigation. Journal of Consulting and Clinical Psychology, 56, 210-217.

Markman, H. J., Renick, M. J., Floyd, F., Stanley, S., y Clements, M. (1993). Preventing marital distress through communication and conflict management training: A four and five year follow-up. Journal of Consulting and Clinical Psychology, 62, 1-8.

Markman, H. J., y Rhoades, G. K. (2012). Relationship Education Research: Current Status and Future Directions. Journal of Marital and Family Therapy, 38, 169-200.

Markman, H. J., Rhodes, G. K., Stanley, S. M., Ragan, E. P., y Whitton, S. W. (2010). The Premarital Communication Roots of Marital Distress and Divorce: The First Five Years of Marriage, Journal of Family Psychology, 24, 289-298.

Markman, H. J., Stanley, S. M., y Blumberg, S. L. (1994). Fighting for Your Marriage: Positive Steps for loving and lasting relationchips. San Francisco: Jossey-Bass.

Markman, H. J., Stanley, S. M., Jenkins, N. J., Petrella, J. N., y Wadsworth, M. E. (2006). Preventive Education: Distinctives and Directions. Journal of Cognitive Psychotherapy: An International Quarterly, 20, 411- 433.

Mathematica Policy Research (2003). Recuperado desde http://www. mathematica-mpr.com/About_Us/

Ministerio del Interior (2010). Primera Encuesta Nacional de Victimización por Violencia Intrafamiliar y Delitos Sexuales del Ministerio del Interior. Departamento de Estudios Sociológicos de la Pontificia Universidad Católica de Chile (DESUC). Santiago: Ministerio del Interior.

Morrison, D. R., y Coiro, M. J. (1999). Parental Conflict and Marital Disruption: Do Children Benefit When High-Conflict Marriages Are Dissolved?. Journal of Marriage and the Family, 6, 626-637.

Office of Planning, Research y Evaluation OPRE (2013). Building Strong Families, 2002-2012. Recuperado desde http://www.acf.hhs.gov/ programs/opre/research/project/building-strong-families-2002-2012

Ooms, T. (2002). Marriage and government: Strange bedfellows? Couples and Marriage Policy Brief No. 1. Washington, DC: Center for Law and Social Policy. Recuperado desde http://www.policyarchive.org/ handle/10207/bitstreams/13927.pdf

Ooms, T. (2005). The new kid on the block: What is marriage education and does it work? (Policy Brief No. 7). Washington, D.C.: Center for Law and Social Policy. Recuperado desde http://www.clasp.org/admin/ site/publications_archive/files/0183.pdf
Ooms, T. (2007). Adapting Healthy Marriage Programs for Disadvantaged and Culturally Diverse Populations: What are the issues?. Center for Law and Social Policy. Couple and Marriage Series. Brief $\mathrm{N}^{\circ} 10$.

Ooms, T., y Wilson, P. (2004). The challenges of offering relationship and marriage education to low-income populations. Family Relations, $53,440-447$.

Petch, J., Halford, W. K.,. Creedy, D. K., y Gamble, J. (2012). Couple Relationship Education at the Transition to Parenthood: A Window of Opportunity to Reach High-Risk Couples. Family Process, 51, 498-511.

Póo, A. M., y Vizcarra, M. B. (2010). Diseño, Implementación y Evaluación de un Programa de prevenión de la Violenia en el Noviazgo. Terapia Psicológica, 29, 213-223.

Renick, M. J., Blumberg, S., y Markman, H. J. (1992). The Prevention and Relationship Enhancement Program (PREP): An empiricallybased preventive intervention program for couples. Family Relations, 41, 141-147.

Rivera, D., Cumsille, P., y Domínguez, C. (2012). Proyecto Diseño, Implementación y Evaluación de un Programa Psicoeducativo de Desarrollo de Habilidades Relacionales para Parejas que contraerán matrimonio. FONDECYT Regular (2012-2014). N 1120940.

Robles, T. F., y Kiecolt-Glaser, J. K. (2003). The physiology of marriage: pathways to health. Physiology y Behavior, 79, 409-416.

Berger, R., y Hannah, M. T. (1999). Preventive Approaches in Couple Therapy.USA: BRUNNER/MAZEL.

Schilling, E. A., Baucom, D. H., Burnett, Ch. K., Allen, E. S., y Ragland, L. (2003). Altering the Course of Marriage: The Effect of PREP Communication Skills Acquisition on Couples' Risk of Becoming Maritally Distressed . Journal of Family Psychology, 17, 41-53.

Silliman, B., y Schumm, W. R. (2000). Marriage Preparation Programs: A Literature Review. The Family Journal: Counseling snd Therapy for Couples and Families, 8, 133-142.

Silliman, B., Stanley, S. M., Coffin, W., Markman, H. J., y Jordan, P. L. (2002). Preventive interventions for couples. En H. A. Liddle, D. A. Santisteban, R. F. Levant, y J. H. Bray (eds), Family psychology: Science-based interventions (pp. 123-146). Washington, DC: American Psychological Association.

Stanley, S. M. (2001). Making the case for premarital training. Family Relations, 50, 272-280.

Stanley, S. M., Amato, P. R., Johnson, C. A., y Markman, H. J. (2006). Premarital education, marital quality, and marital stability: Findings from a large, random, household survey. Journal of Family Psychology, 20,117-126.

Stanley, S. M., Allen, E.S., Markman, H. J., Saiz, C. C., Bloomstrom, G., Walter, R. T., Schumm, R., Bailey, A. E. ( 2005). Dissemination and Evaluation of Marriage Education in the Army. Family Process, 44, 187-201.

Stanley, S. M., Rhoades, G. K., Olmos-Gallo, P. A., y Markman, H. J. (2007). Predicting marital distress from changes in communication following PREP: Findings and methodological concerns. Prevention Science, 8, 227-239.

Troxel, W. M., y Matthews, K. A. (2004). What Are the Costs of Marital Conflict and Dissolution to Children's Physical Health?. Clinical Child and Family Psychology Review, 7, 29-57.

United Nations (2011). 2009-2010. Demographic Yearbook. Department of Economic and Social Affairs. New York: United Nations Publication. Recuperado desde http://unstats.un.org/unsd/demographic/products/ dyb/dybsets/2009-2010.pdf

van Acker, E. (2003). Administering romance: Government policies concerning pre-marriage education programs. Australian Journal of Public Administration, 62, 15-23.

van Acker. E. (2008). Governments and Marriage Education Policy : Perspectives from the UK, Australia and the US. London: Palgrave Macmillan. 